\title{
La enseñanza de la química vista desde la perspectiva de Jensen: Una red conceptual sobre equilibrio químico
}

The teaching of the chemistry seen from the Jensen's perspective: A conceptual net on chemical balance

Ángela Viviana Castrillón Univio, Miguel Angel Rey Villalobos y Yanith Jimenez Rojas

Universidad Pedagógica Nacional. angela castrillonu@yahoo.es, mrey7@hotmail.com, yayisyan@yahoo.es.

\section{Resumen}

Este trabajo propone una red conceptual basado en el concepto generador equilibrio químico, para la enseñanza de la química desde la propuesta epistemológica de Jensen W. B con el objetivo de integrar la historia y la epistemología en la realización posterior de una propuesta de diseño curricular la cual está definida en el marco de la ley general de educación Ley 115 de 1994, para tal propósito se toma como referencia bibliográfica los postulados de epistemólogos reconocidos como Popper, Lakatos y Khun, luego se describe las concepciones de enseñanza-aprendizaje que posee el profesor como producto de su visión de ciencia, finalmente como éstas se articulan, con la propuesta de Jensen $W$. B y en el diseño curricular.

\section{Abstract}

This work proposes a conceptual network based on the chemical equilibrium concepts for the teaching of chemistry since the proposal of epistemological $W$. Jensen B with the objective of integrating the history and epistemology in a subsequent proposal for a curriculum which is defined under the Education Act 1994, Act 115, for this purpose is taken as the principles of bibliographic epistemologist recognized Popper, Lakatos and Kuhn, then describes the conception of teachinglearning has the teacher as a product of his vision of science, as ultimately they are linked with the proposal of $W$. Jensen $B$ and in curriculum design.

Palabras claves

Enseñanza-aprendizaje, currículo, equilibrio químico, estructura lógica, red conceptual.

\section{Keywords}

Teaching-leaming, curriculum, chemical balance, structure, logic, conceptual network.

\section{Introducción}

El siguiente trabajo se realiza con el objetivo estructurar una propuesta para la enseñanza de la química en los grados correspondientes a decimo y once, visto desde la perspectiva de Jensen (1998), explicando el equilibrio químico como tema central siendo este estructurado en una red conceptual, sabiendo que en la educación se contempla la necesidad de un conocimiento científico obligatorio señalado en los objetivos para la educación media según el artículo $\mathbf{3 0}$ de la ley general 115 (MEN,1994), incluido en los estándares básicos de competencias en ciencias naturales que responden espećficamente a la aproximación del estudiante como cientúfico natural, (MEN, 1998), relacionando la estructura de las moléculas orgánicas e inorgánicas con sus propiedades físicas y químicas y su capacidad de cambio químico (MEN, 1998). 
Partiendo de la importancia de generar propuestas en el aula de dase que integren en el diseño aurricular la historia y la epistemología como aspectos del conocimiento cient́fico importantes para la enseñanza de las ciencias, en este sentido se hace necesario realizar un análisis de las concepciones que tiene el profesorado, producto de diversas visiones de ciencia que manejan los mismos a partir del desarrollo epistemológico que ha tenido el concepto de la misma; de igual manera se indaga la idea de curríaulo y sus implicaciones en el desarrollo de la educación.

\section{Desarrollo}

En cuanto a las concepciones epistemológicas de los profesores, en primer lugar se realiza una descripción de la evolución de la ciencia para enfocar las diversas visiones que poseen los profesores, en este sentido y en cuanto a la evolución del conocimiento hablamos de (Popper, 1959), quien afirmaba que las ciencias empíricas son sistemas de teońas; y la lógica del conocimiento cient́fico, por tanto, puede describirse como una teoría de teorías. El planteó una teoría guiado por un modelo falsacionista, este enfoque propone que sean aceptados por convención los enunciados básicos, facticos, singulares en un sentido espacio-temporal, en lugar de las teorías espacio-temporalmente universales. Una teoría es científica solo si puede entrar en conflicto con un enunciado básico, y una teoría debe ser eliminada si entra en conflicto con un enunciado básico aceptado. El mismo explicó que (por el lado positivo): 1.el principal estímulo externo de las teorías científicas procede de la no cient́fica "metafísica" e induso de mitos y que (por el lado negativo): 2 Los hechos no constituyen tales estímulos externos (los descubrimientos facticos íntegramente forman parte de la historia interna constituyendo refutaciones de alguna teoría científica, de modo que los hechos solo son observados si entran en conflicto con algunas expectativas previas) (Lakatos, 1978). Brindando entonces el concepto de teoría como un enunciado universal, que puede ser falseable.

Por otra parte, para Lakatos según su metodología, los grandes logros científicos son programas de investigación que pueden ser evaluados en términos de transformaciones progresivas y regresivas de un problema. La unidad básica para la evaluacion no debe ser una teoría aislada 0 una conjunción de teorías, sino un programa de investigación con un centro firme convencionalmente aceptado (y por tanto, irrefutable por decisión provisional) y con una heurística positiva que define los problemas, esboza la construcción de un cinturón de hipótesis auxiliares, prevé anomalías y las transforma en ejemplos según un plan preconcebido, todo ello. Como aualquier otra metodología, la metodología de los programas de investigación constituye un programa de investigación historiográfico. Pero esta misma traza una demarcación entre historia interna y extema. Esto es, convierte en intemos a muchos problemas que habían sido extemos para otras historiografías. De igual manera, insiste en la duradera rivalidad técnica y empírica de los principales programas de investigación en donde cada reconstrucción racional produce un patrón característico del crecimiento racional del conocimiento científico. Pero todas estas reconstrucciones normativas pueden requerir teorías empíricas externas para explicar los factores residuales no racionales. La historia de la ciencia siempre es más rica en su reconstrucción racional. Pero la reconstrucción racional o historia interna es lo principal; la historia externa es secundaria puesto que los problemas más importante de la historia externa son definidos por la historia intema. (Lakatos, 1978).

Finalmente, se puede resumir la imagen quee tiene Khun de cómo progresa una ciencia mediante el siguiente esquema abierto:

Pre ciencia-ciencia normal- crisis- revolución-nueva ciencia normal. Nueva crisis

La desorganizada y diversa actividad que precede a la formación de una ciencia se estructura y dirige finalmente cuando una comunidad cientŕica se adhiere a solo un paradigma. Un paradigma está constituido por los supuestos teóricos generales, las leyes y las técnicas para su aplicación que 
adoptan los miembros de una determinada comunidad científica (Kuhn, 1962). En donde la ciencia normal según el articulará y desarrollará el paradigma en su intento por explicar y acomodar el comportamiento de algunos aspectos importantes del mundo, definiendo de este modo a ciencia normal como la investigación basada firmemente en uno o más logros científicos pasados, logros que una comunidad científica particular reconoce durante algún tiempo como el fundamento de su práctica ulterior.

Al hacerlo experimentaran inevitablemente dificultades y se encontraran con aparentes falsaciones. Si las dificultades de este tipo se escapan de las manos, se desarrolla un estado de crisis. La crisis se resuelve cuando surge un paradigma completamente nuevo que se gana la adhesión de un número de cientúficos cada vez mayor, hasta que finalmente se abandona el paradigma original, acosado por problemas, es decir, cuando este nuevo paradigma rompe radicalmente con el anterior, se produce una revolución cient́fica. El cambio discontinuo constituye esta misma. De tal manera que la nueva ciencia normal consiste en la realización lograda mediante la ampliación del conocimiento de aquellos hechos que el paradigma muestra como particularmente reveladores, aumentando la extensión del acoplamiento entre estos hechos y las predicciones del paradigma y por medio de la articulación ulterior del paradigma mismo. El nuevo paradigma, lleno de promesas y no abrumado por dificultades en apariencia insuperables, guía entonces la nueva actividad científica normal hasta que choca con serios problemas y aparece una nueva crisis seguida de una nueva revolución.

Actualmente y gracias a las investigaciones en didáctica de las ciencias se reconoce que las distintas concepciones de ciencia que poseen los maestros influyen en sus visiones de enseñanza y aprendizaje (Porlan,1997), constituyéndose estas últimas como cruciales en la práctica profesional mostrando que los modelos tradicionales de enseñanza que son aún los mas recurridos, responden a visiones de la ciencia empiro-inductivista y ateórica (Unesco, 2005), dicha concepción defiende el papel de la observación y de la experimentación "neutras" (no contaminadas por ideas apriorísticas), olvidando el papel esencial de las hipótesis como focalizadoras de la investigación y de los auerpos coherentes de conocimientos (teorías) disponibles, que orientan todo el proceso (Unesco, 2005).

De esta forma, las concepciones de enseñanza y aprendizaje se ven afectadas por las visiones epistemológicas que predominan en los profesores, llevando a elaboraciones curriculares particulares, las auales están contempladas en la definición de currículo entendido según Lundgren (1997) como la solución necesaria al problema de la representación, y el problema de la representación como el objeto del discurso pedagógico. Este objeto del discurso se plantea como un domino de pensamiento y de la constucción de unas realidades sociales especificas cuando la producción social y la reproducción se separan entre sí y particularmente en Colombia y según el Ministerio de educación nacional (MEN, 1994) como "...un conjunto de criterios, planes de estudio, programas, metodologías y procesos que contribuyen a la formación integral y a la construcción de la identidad cultural nacional, regional y local".

En este sentido las elaboraciones curriculares poseen la intención señalada con anterioridad pero su ejecución corresponde a las perspectivas de enseñanza y aprendizaje que el profesor maneje, de esta forma en la tabla 1 se intenta describir y relacionar las concepciones con la práctica en el aula y la construcción curricular. 
Tabla 1. Relaciones entre concepciones de enseñanza de las ciencias y elaboración curricular.

\begin{tabular}{|c|c|c|}
\hline $\begin{array}{l}\text { Concepción de } \\
\text { enseffanza y } \\
\text { aprendizaje }\end{array}$ & $\begin{array}{c}\text { Descripción del trabajo en el aula. (porlan, } \mathbf{r} \\
\text { 1997). }\end{array}$ & $\begin{array}{c}\text { Analisis de categorias acerca de } \\
\text { la elaboración curricular. } \\
\text { (mora, 1999) }\end{array}$ \\
\hline MODELO TRADICIONAL & $\begin{array}{c}\text { MOMENTO } 1 \\
\text { Explicación del profesor. Relato de contenidos y } \\
\text { conceptos siguiendo una lógica formal y académica, } \\
\text { con ayuda ocasional del libro de texto y procurando } \\
\text { que los alumnos comprendan se enteren } \\
\text { MOMENTO } 2 \\
\text { Realización de actividades previstas para fijar los } \\
\text { contenidos. } \\
\text { Generalmente son propuestas sacadas de los libros de } \\
\text { texto y modificadas por el profesor. } \\
\text { La mayor parte de ellas son preguntas que inducen a } \\
\text { producir lo explicado en dase. Se trata de actividades } \\
\text { fundamentalmente mecánicas que refuerzan la } \\
\text { memorización. } \\
\text { MOMENTO } 3\end{array}$ & $\begin{array}{l}\text { Curriculum y programación: } \\
\text { Tendencia al intelectualismo y } \\
\text { enciclopedismo y por ende es } \\
\text { superficial y centrado en dominios } \\
\text { solamente conceptuales. Se } \\
\text { presenta imposibilidad de integrar } \\
\text { distintos tipos de conocimiento. La } \\
\text { secuenciación de contenidos de } \\
\text { temas y de actividades es mecánica, } \\
\text { aditiva, rígida y lineal. La } \\
\text { programación está centrada en } \\
\text { contenidos ya elaborados y } \\
\text { ordenados de acuerdo con la lógica } \\
\text { de la disciplina científica y no de la } \\
\text { mente de los estudiantes (Pedagogía } \\
\text { por Contenidos). } \\
\text { Se presenta una dependencia hacia } \\
\text { el manejo del conocimiento } \\
\text { dependiendo del libro texto } \\
\text { (Textocentrismo). }\end{array}$ \\
\hline MODELO TECNOLOGICO & $\begin{array}{c}\text { MOMENTO } 1 \\
\text { Programación exhaustiva de objetivos generales, } \\
\text { espećficos y operativos. Programación y } \\
\text { temporalización de secuencias cerradas de actividades, } \\
\text { íntimamente relacionada con dichos objetivos. } \\
\text { MOMENTO } 2 \\
\text { Aplicación, lo más fielmente posible, de las secuencias } \\
\text { de actividades programadas. } \\
\text { MOMENTO } 3 \\
\text { Evaluación de los alumnos en relación a los objetivos } \\
\text { operativos. Realización de actividades de recuperación. } \\
\text { Modificación, en su caso, de la programación. }\end{array}$ & $\begin{array}{c}\text { Curricu/um y programación: } \\
\text { Tecnológico. } \\
\text { Los contenidos por enseñar son una } \\
\text { versión de los contenidos } \\
\text { disciplinares secuenciados bajo una } \\
\text { concepción de lo simple (observable) } \\
\text { a lo complejo (abstracto), siendo el } \\
\text { número de conceptos que se } \\
\text { pretende enseñar considerablemente } \\
\text { menor que en las propuestas } \\
\text { anteriores. Se produce una falta de } \\
\text { atención por los contenidos en pro } \\
\text { de la ejecución de experimentos } \\
\text { inconexos. Constituye un modelo de } \\
\text { aprendizaje basado en concepciones } \\
\text { empírico / inductivistas de la ciencia, } \\
\text { en donde no se tiene en auenta la } \\
\text { elaboración de hipótesis y el diseño } \\
\text { de experimentos. Esta visión del } \\
\text { método cient́fico infravalora la } \\
\text { creatividad del trabajo cient́fico } \\
\text { llevando a pensar, a los alumnos, } \\
\text { que la ciencia consiste } \\
\text { en verdades incontrovertibles. }\end{array}$ \\
\hline $\begin{array}{c}\text { MODELO } \\
\text { ESPONTANEISTA. }\end{array}$ & $\begin{array}{c}\text { MOMENTO } 1 \\
\text { Detección de los intereses de los alumnos y elección de } \\
\text { propuestas de trabajo. } \\
\text { MOMENTO } 2\end{array}$ & $\begin{array}{l}\text { Curriculum y programación: } \\
\text { Espontaneísta. } \\
\text { La programación es poco sistemática } \\
\text { y explícita, se improvisan los } \\
\text { contenidos, donde predominan las } \\
\text { actitudes y procedimientos de los } \\
\text { alumnos. } \\
\text { Se produce una falta de atención por } \\
\text { los contenidos en pro de la ejecución } \\
\text { de experimentos inconexos. }\end{array}$ \\
\hline
\end{tabular}


En este marco de ideas se considera que las propuestas de integración curricular relacionadas con las concepciones de enseñanza y aprendizaje anteriores no enfatizan en el papel de la historia y la epistemología en el desarrollo del conocimiento cient́fico en la enseñanza de las ciencias, además son estos mismos modelos los más utilizados dentro del aula de dase por parte de los profesores sin tener en cuenta el papel que cumplen los modelos alternativos, los cuales se han venido investigando desde la década de los ochenta; estos son dirigidos desde un punto de vista epistemológico tendientes a proveer una enseñanza de las ciencias acorde con un entendimiento de la metodología cientúfica; estos modelos de corte constructivistas asumen el desempeño docente en relación con la existencia de un curriculum oculto que determina la enseñanza de los alumnos en donde la epistemología del profesor afecta el modo en que se enfoca el proceso de enseñanza (¿???) por tal motivo, se retoma la perspectiva de Jensen quien considera que el estudio de la química proporciona un prototipo de cómo organizar lógicamente conceptos y modelos de química, a partir de la pregunta que él plantea citiene la química una estructura lógica? Realizando un esquema de clasificación a partir de su visión de cómo podría parecer la estructura lógica de la misma. Dividiendo los conceptos y los modelos de la química dentro de tres categorías o dimensiones basados en si ellos se tratan de composición/estructura con energía, o con el rol de tiempo en procesos químicos. Cada una de estas dimensiones puede, a su vez, acercarse a uno de los tres niveles conceptuales el molar, el molecular o el eléctrico donde cada uno corresponde históricamente a una revolución química mayor (Jensen, 1998).

Tabla No2 La estructura lógica de la química.

\begin{tabular}{|c|c|c|c|}
\hline & $\begin{array}{c}\text { Dimensión } \\
\text { Composición y estructura }\end{array}$ & $\begin{array}{l}\text { Dimensión } \\
\text { Energía }\end{array}$ & Dimensión tiempo \\
\hline Nivel molar & $\begin{array}{l}\text { 1. composición simple relativa y } \\
\text { sustancias compuestos puros, soluciones y } \\
\text { mezdas. Designación empírica de } \\
\text { alomorfos (estado, color, forma } \\
\text { cristalográfica, a, } \beta \text {, etc. }\end{array}$ & $\begin{array}{l}\text { 4.calorimetria, entropía y } \\
\text { calor de formación, energía } \\
\text { libre y constantes de } \\
\text { equilibrio }\end{array}$ & $\begin{array}{l}\text { 7. leyes, proporciones } \\
\text { experimentales. parámetros } \\
\text { experimentales, Arrhenius y } \\
\text { I o entropía y calores de } \\
\text { activación }\end{array}$ \\
\hline Nivel molecular & $\begin{array}{l}\text { 2. formulas estructurales y absolutas. } \\
\text { Racionalización de alomorfos como } \\
\text { variaciones en composiciones absolutas } \\
\text { (polímeros) o estructuras (isómeros). }\end{array}$ & $\begin{array}{l}\text { 5. interpretación molecular } \\
\text { de entropía. Interpretación } \\
\text { de calores de formación en } \\
\text { términos de calores de } \\
\text { atomización, el promedio de } \\
\text { energías de enlace, etc. } \\
\text { Mecánica molecular }\end{array}$ & $\begin{array}{l}8 . \quad \text { mecanismos de } \\
\text { reacciones moleculares vita } \\
\text { molecular de entropías de } \\
\text { activación y complejos } \\
\text { activados }\end{array}$ \\
\hline $\begin{array}{l}\text { Nivel } \\
\text { eléctrico }\end{array}$ & $\begin{array}{l}\text { 3. Formulas electrónicas (Lewis str. Y } \\
\text { configuración electrónica). Variaciones...o } \\
\text { composición nudear (iones e isotopos) o } \\
\text { estructura (estados excitados) }\end{array}$ & $\begin{array}{l}\text { 6. calculo de energías } \\
\text { basadas en la estructura } \\
\text { electrónica. Interpretación } \\
\text { de espectros. Calculo de } \\
\text { calores de atomización, } \\
\text { entropías espectroscópicas, } \\
\text { etc. }\end{array}$ & $\begin{array}{l}\text { 9. mecanismos de } \\
\text { reacciones iónicas y } \\
\text { fotoquímicas, } \\
\text { isotópicos. Calcectos } \\
\text { energías de activación. } \\
\text { Índices de reactividad } \\
\text { electrónica. }\end{array}$ \\
\hline
\end{tabular}

Dentro de la propuesta de Jensen la estructura lógica de la química se desarrolla en tres revoluciones señalando la importancia de la historia para el abordaje de la química. Estas tres revoluciones están enmarcadas en un periodo histórico determinado por la evolución del conocimiento químico, la primer revolución química descrita entre 1770 y 1790, relaciona a los factores que contribuyeron a la química desde la química neumática, la teoría de la combustión y la respiración, la teoría de calcinación, la teoría de causticidad y la teoría calónica de los estados; la segunda revolución química comprendida desde 1855 hasta 1875, basada en el atomismo especulativo y la composición gravimétrica dentro de la teoría atómica, de igual manera se toma en cuenta la taxonomía orgánica, los calores espeáficos, las densidades de los gases y la composición volumétrica y por último la tercera revolución química extendida desde 1904 hasta1924 la que abarca desde las hipótesis cuánticas, la tabla periódica, la electroquímica, radioactividad, tubos de descarga hasta la espectrofotometría. 
A partir de esta perspectiva estructuramos un diseño auriaular, que toma como concepto generador y al que categorizamos es el equilibrio químico, ya que su construcción epistemológica permite interrelacionar conceptos espeáficos en química logrando una estructura lógica configurándolo en una red conceptual, la cual según (Vasco, Bermúdez, Escobedo y León, 2000) consideran que una red de enunciados y proposiciones armadas con ciertas combinaciones de conceptos y relaciones configuran el subsitema dedarativo de la disciplina, de quimica en este caso, que le permite dedarar teorias explicativas sobre los problemas enfocados por dicha disciplina, en este sentido la red conceptual es complejizada en la medida en que interrelaciona los conceptos.

En este orden de ideas, definimos conceptos que se interelacionan en la red conceptual equilibrio quimico, partiendo de las dimensiones que señala Jensen, como se muestra en la siguiente tabla:

\begin{tabular}{|l|l|l|l|}
\hline Niveles/dimensiones & \multicolumn{1}{|c|}{ Estructural } & \multicolumn{1}{|c|}{ Energética } & \multicolumn{1}{|c|}{ Temporal } \\
\hline molar & $\begin{array}{l}\text { Ácidos y bases } \\
\text { Reacciones químicas } \\
\text { Titulación, } \\
\text { Concentraciones }\end{array}$ & enlaces & Bronsted y Lowry \\
\hline molecular & $\begin{array}{l}\text { Elementos, } \\
\text { periodicidad, } \\
\text { Nomendatura química, }\end{array}$ & Iones, moleculas & $\begin{array}{l}\text { Gilbert Lewis, Dimitry } \\
\text { Mendeliev, Berzelius, } \\
\text { Lothar mayer }\end{array}$ \\
\hline $\begin{array}{l}\text { Configuración } \\
\text { electrónica }\end{array}$ & Niveles, subniveles & Erwin Schrödinger \\
\hline
\end{tabular}

\section{Apreciaciones finales}

De esta forma, se comparte la perspectiva de Jensen en donde la química puede y tiene una estructura lógica con unos niveles que jerarquizan el cuerpo conceptual de la misma, aunque dichos conceptos mantienen los niveles organizacionales se acomodan a las diferentes dimensiones descritas con anterioridad, como por ejemplo el concepto "reacciones químicas"; el aual varia dentro de las dimensiones dependiendo del punto de vista con el que se trabaje, si hablamos de la dimensión energética podemos referimos a las reacciones químicas como endotérmicas y/o exotérmicas pero si se refiere a reacciones químicas como dimensión estructural nos referimos al tipo de reacción que puede ser de síntesis o de descomposición.

Además, cabe resaltar que los educadores deben evidenciar la importancia que tiene la epistemología dentro de la estructura lógica de la química, para que de esta manera se contribuya con los procesos de enseñanza-aprendizaje en el marco de la integración curricular tanto para el educando como en el educador.

Para integrar en el currículo la epistemología y la historia de la química, es necesario que el profesor reflexione frente a su práctica profesional, sabiendo si su versión de ciencia coincide con sus concepciones de enseñanza-aprendizaje, y esta como se refleja en el diseño curricular.

\section{Bibliografía} 689.

Jensen, W. (1998). Does chemistry have a logical structure? joumal of chemical education, 679-

Jensen, W. (1998). Logic, history, and the chemistry textbook. joumal of chemical education, 817-829.

Khun, T. (1962). la estructura de las revolucioneus cientificas. Mexico: Fondo de cultura economica.

Lakatos, I (1978). la metodologia de los programas de investigacion cientifica. Madrid: Alianza editorial.

MEN. (1994). Ley General de educacion. Bogota: Magisterio.

Popper, K. (1934). La logica de la investigación cientúfica. 